\title{
Polycyclic Aromatic Hydrocarbons in Soil Adjacent to Highways in Beijing, People's Republic of China
}

\author{
S.-G. Chu, H. Liu, L.-L. Ma, X.-B. Xu \\ Research Center for Eco-Environmental Sciences, Chinese Academy of Sciences, \\ Beijing 100085, People's Republic of China
}

Received: 15 July 2002/Accepted: 1 February 2003

The industrial revolution marked the onset of a major jump in traffic capacities and both our economy as well as social well-being are heavily dependent on the mobility today. All positive effects of our current mobility aside, negative impacts of traffic on environment quality have increased considerably now. We are currently faced with more and more serious new problems such as traffic noise and exhaust emissions. Most of investigations showed that traffic, especially highway, is the major pollution source in city and industrial areas. In roadside environmental investigations, while the concentration of carbon monoxide, oxides of nitrogen and others in air near highway are monitored, and many studies have been done on heavy metals such as $\mathrm{Pb}, \mathrm{Cr}$, and $\mathrm{Cd}$, little attention has been paid to the contamination of hydrocarbon pollutants, such as polycyclic aromatic hydrocarbons (PAHs). Polycyclic aromatic hydrocarbons (PAHs) are one of the most common groups of environmental contaminants (Wilcke 2000). PAHs are globally distributed and the highest concentrations generally occur close to urban centers (Yang 2002). The adverse effects of these compounds on human health have been well documented. They include genotoxicity and carcinogenity (Perera 1997; Yang and Silverman 1988).

Motor vehicle exhaust emissions into air and release some PAHs into roadside environment as a result of incomplete combustion in their engines. Analyses of sediment collected from reservoir near highway indicated that they contained elevated levels of PAHs, and presumably, motor vehicle was a major source of these contaminants (Ishimaru et al 1990). Due to their low vapor pressure, most of PAHs immediately condense and adsorb onto particulates after they are released from engine, which will further deposit on earth as a part of soil, or they may directly adsorb on soil. There is concern about polycyclic aromatic hydrocarbons, as high values have been reported in roadside soil (Butler et al 1984).

Although traffic is mainly dependent on railway in China, more and more highways are being built in China now to meet the needs of economic development. Highway building becomes one of key projects of infrastructure development in China now. There is an urgent need to investigate the impact of highway building to environmental system. The objective of the present study was to investigate the pollution of PAHs in soil along typical highways in China. 


\section{MATERIALS AND METHODS}

The study sites (A, B, C, and D) located on the four main highways in the outskirts of Beijing City (Figure 1). Highway A is Jing-Chang highway, which is located in the north of the city, and it is the main road connecting Beijing City with the main view spot of the Great Wall. Highway B is Beijing Capital Airport Expressway, which located in the north-east of the city, it was rebuilt three year ago and the main vehicles are cars. Highway $\mathrm{C}$ is Jing-Jin highway, which is the main highway connecting Beijing City with Tianjin City, which is another main industrial city in the north of China. Highway D was Jing-Shi highway and it is the earliest expressway near Beijing City, which was built more than fifteen years ago. The sample areas were parts of outskirts of Beijing City with high traffic density. All the sample sites were fully covered with herbal vegetation except for samples collected near road verge. To compare the contamination of PAHs polluted by highway traffic, a cultivated soil sample site, which was far from traffic road, was chosen and sampled at the same time. The sample, named as E, can be regarded as reference sample from an unaffected landscape. The soils were sampled in May 2001. Soil was sampled with a coring cylinder along four parallel lines of highway, at distances of $0.2 \mathrm{~m}, 10 \mathrm{~m}, 30 \mathrm{~m}$ and $50 \mathrm{~m}$ from the asphalt surface. For highway A, one more sample was taken which located parallel with highway at $70 \mathrm{~m}$. The upper $10 \mathrm{~cm}$ soil was sampled in 20 locations along each line. The soil samples from the roadside were individually ground and mixed thoroughly. The remaining water in soil was measured by drying subsample in oven at $105^{\circ} \mathrm{C}$ for $12 \mathrm{~h}$, and determined gravimetrically. All the results were reported as dried weight base.

The 16 PAHs standards, naphthalene, acenaphthylene, acenaphthene, fluorene, phenanthrene, anthracene, fluoranthene, pyrene, benz(a)anthracene, chrysene, benzo(b)fluoranthene, benzo(k)fluoranthene, benzo(a)pyrene, indeno(123cd)pyrene, dibenz(a,h)anthracene, and benzo(ghi)perylene, were obtained from Aldrich (WIS, USA). 2-Flurobiphenyl was purchased from Supelco (Bellefonte, PA, USA). All the solvents used were of analytical grade, obtained from Beijing Chemical Factory (Beijing, China). The solvent was redistilled in all glass system before use. Silica gel (100-200 mesh) was obtained from Qingdao Haiyang Chemical Co. (Shandong, China) and activated at $130^{\circ} \mathrm{C}$ for $12 \mathrm{~h}$, then kept it in a desiccator.

About $5 \mathrm{~g}$ soil was accurately weighed and mixed with anhydrous sodium sulfate. After $1 \mathrm{~mL}$ of 2-florobuphenyl solution $(0.2 \mu \mathrm{g} / \mathrm{mL})$ was added as internal standard, the sample was ultrasonically extracted with $30 \mathrm{~mL}$ of acetone/ hexane $(1: 1)$ for 5 minutes. The soil and extract were separated by centrifugation and the extraction process was repeated three times. The combined extracts were concentrated by $K$. D. evaporative concentrator and the solvent changed to hexane. The fractionation was performed by column chromatograph. $10 \mathrm{~g}$ silica was weighed in beaker and dichloromethane was added to make a silica gel slurry, and then it was used to charge the column $(400 \mathrm{~mm} \times 10 \mathrm{~mm}$ i.d.). About $1 \mathrm{~g}$ anhydrous sodium sulfate was added on the top of the silica gel. The column was 


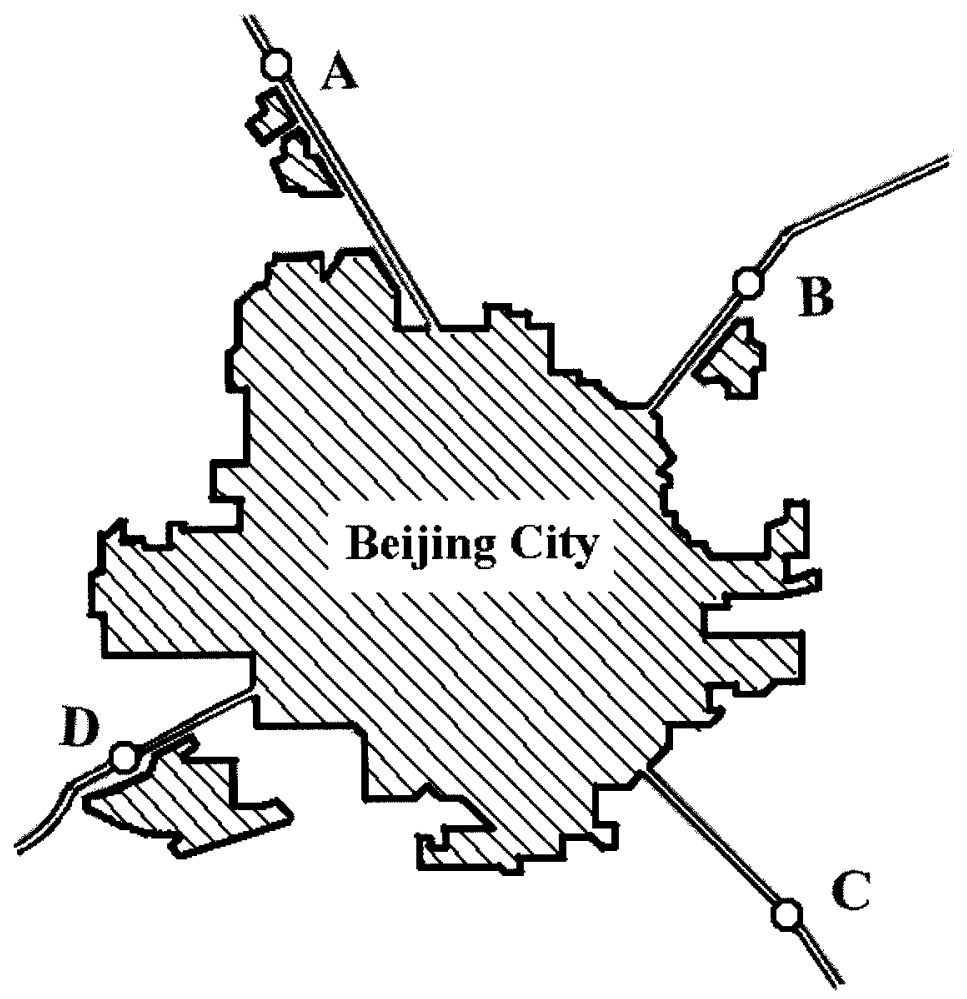

Figure 1. Location of the four soil sampling sites.

pre-eluted with $40 \mathrm{ml}$ of hexane before loading the sample. After the sample was transferred to the column the silica gel column was eluted with $30 \mathrm{~mL}$ of hexane, this fraction containing all the alkane, then eluted with $25 \mathrm{ml}$ hexane/dichoromethane $(15 \mathrm{ml} / 10 \mathrm{ml})$ and this fraction containing all the PAHs. The PAHs fraction was concentrated by $\mathrm{K}$. D evaporative concentrator, and then the solution volume was reduced to $0.2 \mathrm{~mL}$ by a gentle steam of nitrogen.

GC/FID was used to determine PAHs, and the result was confirmed by GC/MSD. A Hewlett-Packard Model 5890-II gas chromatograph equipped with DB-5 column $(30 \mathrm{~m} \times 0,25 \mathrm{~mm}$ with $0.25 \mathrm{~mm}$ with $0.25 \mu \mathrm{m}$ film thickness), was used for the analysis of PAHs. GC oven temperature was initially held for $2 \mathrm{~min}$ at 50 ${ }^{\circ} \mathrm{C}$, increased by $4{ }^{\circ} \mathrm{C} / \mathrm{min}$ to $280^{\circ} \mathrm{C}$, and then held for $20 \mathrm{~min}$. $1 \mu \mathrm{L}$ sample was injected in splitless mode with injector and detector temperature of $280{ }^{\circ} \mathrm{C}$. Identification was based on matching retention times. To confirm the peak identification, some of the samples were also analyzed by GC/MSD. A Hewlett Parkard $6890 \mathrm{GC} / 5973 \mathrm{MSD}$ was used for the confirmation. The GC parameter was similar with that described above, except the carrier gas was helium. The mass spectrometer was operated in scan mode and electron ionization mode 
(70 ev). The recovery was done by spiked known concentration solution of PAHs in uncontaminated soil at the concentration of $10 \mu \mathrm{g} / \mathrm{g}$ level, the recoveries for individual compound ranged from $91 \%$ to $120 \%$. The concentration of those to produce a signal-to-noise of 3:1 in blank sample was defined as limit of detection (LOD). The LOD for PAHs ranged from $0.002 \mu \mathrm{g} / \mathrm{g}$ (chrysene) to $0.04 \mu \mathrm{g} / \mathrm{g}$ (naphthalene). The method was used to analyze PAH contaminated soil/sediment certified reference material CR912 (U.S.EPA). All the measurement fell within the given performance interval.

\section{RESULTS AND DISCUSSION}

Table 1 gives the concentration of all the 16 PAHs determined in the soil samples along highway roadside. To compare the level of contamination, one cultivated soils sample (sample E), which located more than $500 \mathrm{~m}$ from road, was taken and analyzed. The result is also listed in the Table 1. A wide range of aromatic hydrocarbons were found in the soil samples on the roadsides, which included those PAHs that are considered as carcinogenis by the US Environmental Protection Agency, namely benz(a)anthracene, chrysene, benzo(b)fluoranthene, benzo(k)fluoranthene, benzo(a)pyrene, benzo(ghi)perylene, and indeno(123cd)pyrene. Although the concentrations of PAHs vary from the distance of the sample location and also may be effected by other human activity, i. c. rebuilding the road or planting tree alone roadside, the concentration of PAHs in the roadside soil was significantly higher than those of uncontaminated soil.

The concentration of 16 PAHs ranged from 1.47 to $6.61 \mathrm{mg} / \mathrm{kg}$ with the distance from $70 \mathrm{~m}$ to $0.2 \mathrm{~m}$ from roadside of highway $\mathrm{A}$. For most of the samples from distance of $50 \mathrm{~m}$ to $0.2 \mathrm{~m}$, the total concentration of 16 PAHs increased 3 to 5 times. The PAH concentration sharply decreases within the first $10 \mathrm{~m}$, while a substantial slower decrease continues beyond this distance. It was also reported that PAH compounds were mainly transported by air and most of the highwayderived PAH was deposited within $3.8 \mathrm{~m}$ from the road (Johnston and Harrison 1984).

Tuhackova reported that the proximity of a busy highway increased the amount of PAHs in soil at the depth of $5-15 \mathrm{~cm}$ from $106 \mathrm{ng} / \mathrm{g}$ as a grassland background to $3095 \mathrm{ng} / \mathrm{g}$ at highway verge for the 10 determined species in Czech Republic (Tuhackova et al 2001). In our investigation, the total concentration of 16 PAHs in the roadside soil ranged from 1.2 to $2.87 \mathrm{mg} / \mathrm{kg}$. It seems that our data was in satisfactory agreement with those data. It should be mentioned that the total PAHs content was much higher than this level. Besides of the 16 parent PAHs, there are many alkylated-PAHs in samples. If we estimate that the response of alkylatedPAHs in MSD is as same as those of parent PAHs, the total concentration of PAHs in the roadside soil may range from 5 to $12 \mathrm{mg} / \mathrm{kg}$. It was reported that the PAHs content usually ranges from 1000 to $3000 \mu \mathrm{g} / \mathrm{kg}$ in industrial area soil (Weiss et al 1994; Meharg et al 1998). It means that PAHs contamination on roadside soil is quite serious and their contents may even be higher than those of normal industry area. 
Table 1. The concentration of PAHs in roadside soil $(\mathrm{mg} / \mathrm{kg})$

\begin{tabular}{|c|c|c|c|c|c|}
\hline Compound & $0.2 \mathrm{~m}$ & $10 \mathrm{~m}$ & $30 \mathrm{~m}$ & $50 \mathrm{~m}$ & Sample E \\
\hline $\mathrm{NaP}$ & $\frac{(0.13-0.37)}{0.2}$ & $\frac{(0.02-0.17)}{0.11}$ & $\frac{(0.12-0.27)}{0.17}$ & $\frac{(0.10-0.14)}{0.12}$ & 0.03 \\
\hline AcNPy & $\frac{(0.02-0.15)}{0.07}$ & $\frac{(0.02-0.10)}{0.05}$ & $\frac{(0.03-0.09)}{0.05}$ & $\frac{(0.02-0.03)}{0.03}$ & 0.01 \\
\hline $\mathrm{AcNPe}$ & $\frac{(0.01-0.09)}{0.04}$ & $\frac{(0.01-0.06)}{0.02}$ & $\frac{(0.01-0.05)}{0.03}$ & $\frac{(0.01-0.02)}{0.01}$ & ND \\
\hline $\mathrm{FL}$ & $\frac{(0.03-0.15)}{0.09}$ & $\frac{(0.03-0.09)}{0.06}$ & $\frac{(0.02-0.08)}{0.05}$ & $\frac{(0.02-0.04)}{0.03}$ & 0.01 \\
\hline PhA & $\frac{(0.07-0.64)}{0.36}$ & $\frac{(0.10-0.37)}{0.19}$ & $\frac{(0.11-0.34)}{0.23}$ & $\frac{(0.11-0.18)}{0.14}$ & 0.05 \\
\hline AN & $\frac{(0.01-0.16)}{0.09}$ & $\frac{(0.02-0.06)}{0.04}$ & $\frac{(0.02-0.07)}{0.04}$ & $\frac{(0.02-0.04)}{0.03}$ & 0.01 \\
\hline FIA & $\frac{(0.03-0.66)}{0.32}$ & $\frac{(0.09-0.37)}{0.19}$ & $\frac{(0.10-0.46)}{0.22}$ & $\frac{(0.08-0.27)}{0.19}$ & 0.06 \\
\hline Py & $\frac{(0.02-0.46)}{0.25}$ & $\frac{(0.06-0.24)}{0.14}$ & $\begin{array}{c}(0.07-0.40) \\
0.18\end{array}$ & $\frac{(0.06-0.20)}{0.15}$ & 0.03 \\
\hline$B(a) A$ & $\frac{(\text { ND-0.60) }}{0.19}$ & $\frac{(0.02-0.31)}{0.12}$ & $\frac{(0.02-0.18)}{0.08}$ & $\frac{(N D-0.15)}{0.07}$ & 0.02 \\
\hline Chy & $\frac{(0.02-0.60)}{0.22}$ & $\frac{(0.05-0.37)}{0.16}$ & $\frac{(0.05-0.29)}{0.13}$ & $\frac{(0.02-0.19)}{0.1}$ & 0.03 \\
\hline$B(b) F$ & $\frac{(0.02-0.63)}{0.29}$ & $\frac{(0.04-0.47)}{0.18}$ & $\frac{(\text { ND-0.40) }}{0.15}$ & $\frac{(\text { ND-0.23) }}{0.11}$ & 0.06 \\
\hline $\mathrm{B}(\mathrm{k}) \mathrm{F}$ & $\frac{(\text { ND-0.59) }}{0.15}$ & $\frac{(\text { ND-0.36) }}{0.09}$ & $\frac{(\text { ND-0.11) }}{0.04}$ & $\frac{(\text { ND-0.10) }}{0.04}$ & ND \\
\hline$B(a) P$ & $\frac{(N D-0.51)}{0.19}$ & $\frac{(\text { ND-0.25) }}{0.08}$ & $\frac{(\text { ND-0.15) }}{0.07}$ & $\frac{(0.04-0.15)}{0.09}$ & 0.02 \\
\hline $\ln (\mathrm{cd}) \mathrm{P}$ & $\frac{(\mathrm{ND}-0.41)}{0.16}$ & $\frac{(0.01-0.17)}{0.07}$ & $\frac{(0.01-0.08)}{0.04}$ & $\frac{(0.01-0.08)}{0.04}$ & 0.04 \\
\hline $\mathrm{DiB}(\mathrm{ah}) \mathrm{A}$ & $\frac{(\mathrm{ND}-0.10)}{0.05}$ & $\frac{(\text { ND-0.06) }}{0.03}$ & $\frac{(0.01-0.05)}{0.03}$ & $\frac{(0.01-0.03)}{0.02}$ & 0.03 \\
\hline$B($ gih $) P$ & $\frac{(0.01-0.50)}{0.2}$ & $\frac{(0.02-0.29)}{0.12}$ & $\frac{(\text { ND-0.13) }}{0.07}$ & $\begin{array}{c}(0.01-0.14) \\
0.07\end{array}$ & 0.03 \\
\hline$\Sigma$ PAHs & $\frac{(0.39-6.61)}{2.87}$ & $\frac{(0.80-3.59)}{1.63}$ & $\frac{(0.70-2.77)}{1.57}$ & $\frac{(0.67-1.79)}{1.2}$ & 0.43 \\
\hline
\end{tabular}

* Values in parentheses denote range

naphthalene(NaP), acenaphthylene(AcNPy), acenaphthene(AcNPe), fluorene(FL), phenanthrene(PhA), anthracene(AN), fluoranthene(FlA), pyrene(Py), benz(a)anthracene(B(a)A), chrysene(Chy), benzo(b)fluoranthene(B(b)F), benzo(k)fluoranthene $(B(k) F)$, benzo(a)pyrene(B(a)P), indeno(1,2,3-cd)pyrene(I(cd)P), dibenz(a,h)anthracene(DiB(ah)A), benzo(g,h,i)perylene(B(gih)P) 
Acknowledgments. We acknowledge financial support of this work by Major State Basic Research Program of China (No. G1999045707) and KIP of CAS (RCEES 9902).

\section{REFERENCES}

Butler JD, Butterworth V, Kellow SC, Robinson HG (1984) Some observations on the polycyclic aromatic hydrocarbon $(\mathrm{PAH})$ content of surface soils in urban area. Sci Tot Environ 33:75-85

Goodfellow FJL, Murray VSG, Ouki SK, Iversen A, Sparks A, Bartlett T (2001) Public health response to an incident of secondary chemical contamination at a beach in the United Kingdom. Occup Environ Med 58:232-238

Ishimaru T, Inouye H, Morioka T (1990) Risk assessment of drinking water in a reservoir contaminated by PAHs originated from road traffic. Sci Tot Environ 93:125-130

Johnston WR, Harrison RM.(1984) Deposition of metallic and organic pollutants alongside the M6 Motorway. Sci Tot Environ 33:119-127

Meharg AA, Wright J, Dyke H, Osborn D (1998) Polycyclic aromatic hydrocarbon $(\mathrm{PAH})$ dispersion and deposition to vegetation and soil following a large scale chemical fire. Environ Pollut 99:29-36

Perera FP (1997) Environment and cancer: Who are susceptible? Science 278:1068-1073.

Scheepers PTJ, Bos RP (1992) Combustion of diesel fuel from a toxicological perspective II toxicity. Int Arch Occ Environ Hea 64:163-177

Tuhackova T, Cajthaml T, Novak K, Novotny C, Mertelik J (2001) Hydrocarbon deposition and soil microflora as affected by highway traffic. Environ Pollut 113:255-262

Weiss P, Riss A, Gschmeidler E, Schentz H (1994) Investigation of heavy metal, $\mathrm{PAH}, \mathrm{PCB}$ patters and PCDD/F profiles of soil samples from an industrialized urban area (Linz, Upper Austria) with multivariate statistical methods. Chemosphere 29:2223-2236

Yang SK, Silverman BD (1988) Polycyclic aromatic hydrocarbon carcinogenesis: structure activity relationship. CRC Press, Boca Raton, Fla.

Yang Y, Zhang XX, Korenaga T (2002) Distribution of polynuclear aromatic hydrocarbons (PAHs) in the soil of Tokushima, Japan. Water Air Soil Pollut 138:51-60 